\title{
PROBING THE INTERSTELLAR MEDIUM AND STAR FORMATION OF THE MOST LUMINOUS QUASAR AT $z=6.3$
}

\author{
Ran Wang $^{1}$, Xue-Bing Wu ${ }^{1,2}$, Roberto Neri ${ }^{3}$, Xiaohui Fan $^{1,4}$, Fabian Walter ${ }^{5}$, Chris L. Carilli ${ }^{6}$, Emmanuel Momjian ${ }^{6}$, \\ Frank Bertoldi ${ }^{7}$, Michael A. Strauss ${ }^{8}$, Qiong Li $^{2}$, Feige Wang ${ }^{2}$, Dominik A. Riechers ${ }^{9}$, Linhua Jiang ${ }^{1}$, Alain Omont ${ }^{10}$, \\ JEFF WAGG ${ }^{11}$, AND PIERRE Cox ${ }^{12}$ \\ ${ }^{1}$ Kavli Institute of Astronomy and Astrophysics at Peking University, No. 5 Yiheyuan Road, Haidian District, Beijing, 100871, China \\ ${ }^{2}$ Department of Astronomy, School of Physics, Peking University, No. 5 Yiheyuan Road, Haidian District, Beijing, 100871, China \\ ${ }^{3}$ Institute de Radioastronomie Millimetrique, St. Martin d'Heres, F-38406, France \\ ${ }^{4}$ Steward Observatory, University of Arizona, 933 N. Cherry Avenue, Tucson, AZ 85721, USA \\ 5 Max-Planck-Institute for Astronomy, Königsstuhl 17, D-69117 Heidelberg, Germany \\ ${ }^{6}$ National Radio Astronomy Observatory, P.O. Box 0, Socorro, NM 87801, USA \\ ${ }^{7}$ Argelander-Institut für Astronomie, University of Bonn, Auf dem Hügel 71, D-53121 Bonn, Germany \\ ${ }^{8}$ Department of Astrophysical Sciences, Princeton University, Princeton, NJ 08544, USA \\ ${ }_{9}^{9}$ Astronomy Department, Cornell University, 220 Space Sciences Building, Ithaca, NY 14853, USA \\ ${ }^{10}$ Institut d'Astrophysique de Paris, UMR 7095, CNRS and Universite Pierre et Marie Curie, Paris, France \\ ${ }^{11}$ Square Kilometre Array Organisation, Jodrell Bank Observatory, Jodrell Bank, Macclesfield SK11 9DL, UK \\ ${ }^{12}$ Joint ALMA Observatory, Alonso de Córdova 3107, Vitacura, Santiago, Chile \\ Received 2016 May 16; accepted 2016 June 27; published 2016 October 10
}

\begin{abstract}
We report new IRAM/PdBI, JCMT/SCUBA-2, and VLA observations of the ultraluminous quasar SDSS $\mathrm{J} 010013.02+280225.8$ (hereafter, $\mathrm{J} 0100+2802$ ) at $z=6.3$, which hosts the most massive supermassive black hole $(\mathrm{SMBH}), 1.24 \times 10^{10} M_{\odot}$, that is known at $z>6$. We detect the [C II] $158 \mu \mathrm{m}$ fine structure line and molecular $\mathrm{CO}(6-5)$ line and continuum emission at 353,260 , and $3 \mathrm{GHz}$ from this quasar. The $\mathrm{CO}(2-1)$ line and the underlying continuum at $32 \mathrm{GHz}$ are also marginally detected. The [C II] and $\mathrm{CO}$ detections suggest active star formation and highly excited molecular gas in the quasar host galaxy. The redshift determined with the [C II] and CO lines shows a velocity offset of $\sim 1000 \mathrm{~km} \mathrm{~s}^{-1}$ from that measured with the quasar Mg II line. The CO (2-1) line luminosity provides a direct constraint on the molecular gas mass, which is about $(1.0 \pm 0.3) \times 10^{10} M_{\odot}$. We estimate the FIR luminosity to be $(3.5 \pm 0.7) \times 10^{12} L_{\odot}$, and the UV-to-FIR spectral energy distribution of J0100 +2802 is consistent with the templates of the local optically luminous quasars. The derived [C II]-to-FIR luminosity ratio of $\mathrm{J} 0100+2802$ is $0.0010 \pm 0.0002$, which is slightly higher than the values of the most FIR luminous quasars at $z \sim 6$. We investigate the constraint on the host galaxy dynamical mass of $\mathrm{J} 0100+2802$ based on the [C II] line spectrum. It is likely that this ultraluminous quasar lies above the local SMBH-galaxy mass relationship, unless we are viewing the system at a small inclination angle.
\end{abstract}

Key words: galaxies: evolution - galaxies: high-redshift - galaxies: starburst - quasars: individual (SDSS J010013.02+280225.8)

\section{INTRODUCTION}

An ultraluminous quasar, SDSS J010013.02+280225.8 (hereafter, J0100+2802), with a bolometric luminosity of $L_{\text {bol }}=4.3 \times 10^{14} L_{\odot}$ and a supermassive black hole $(\mathrm{SMBH})$ mass of $M_{\mathrm{BH}} \approx 1.2 \times 10^{10} M_{\odot}$, was discovered at $z=6.3$ (Wu et al. 2015). This is by far the most optically luminous object, with the most massive SMBH among the $~ 50$ quasars known at $z>6$ (Fan et al. 2006; Willott et al. 2010; Bañados et al. 2014; Jiang et al. 2015; Venemans et al. 2015; Matsuoka et al. 2016), and the SMBH mass is also comparable to the highest value found in the local universe (McConnell et al. 2011; Thomas 2016). It demonstrates that such a rare, $10^{10} M_{\odot} \mathrm{SMBH}$, could be formed as early as $z \gtrsim 6.3$, when the age of the universe was only $890 \mathrm{Myr}$.

Recent submillimeter and millimeter [(sub)mm] surveys detected strong far-infrared (FIR) continuum, molecular CO, and [C II] $158 \mu \mathrm{m}$ fine structure line emission in the host galaxies of a number of quasars at $z>5.7$ (e.g., Bertoldi et al. 2003; Priddey et al. 2003; Robson et al. 2004; Walter et al. 2004; Carilli et al. 2007; Wang et al. 2010, 2013; Omont et al. 2013; Willott et al. 2015; Venemans et al. 2016). The results argue for significant host galaxy evolution with active star formation in these young quasars in the early universe, and the [C II] line images at sub-second resolution constrain the radius of the nuclear starburst region to be a few kiloparsecs (Leipski et al. 2013; Wang et al. 2013; Venemans et al. 2016). The gas dynamics measured with the $\mathrm{CO}$ and [C II] observations suggest SMBH-host mass ratios of a factor of a few to one order of magnitude higher than the present-day value (Walter et al. 2004; Wang et al. 2013; Willott et al. 2015; Venemans et al. 2016).

The most massive $z>6$ quasar-galaxy systems studied in previous millimeter and radio observations are objects with SMBH masses of a few $10^{9} M_{\odot}$ and $L_{\text {bol }} \lesssim 10^{14} L_{\odot}$ (e.g., Wang et al. 2013; Willott et al. 2015; Venemans et al. 2016). The discovery of $\mathrm{J} 0100+2802$ provides a unique chance to study the quasar-galaxy co-evolution in the system that hosts the most massive known SMBH at the earliest epoch. In this work, we report our new observations of the (sub)mm and radio continuum, [C II] $158 \mu \mathrm{m}$, and molecular CO lines from the host galaxy of $\mathrm{J} 0100+2802$. The observations are described in Section 2, and the results are presented in Section 3. We discuss the quasar spectral energy distribution (SED), FIR continuum, [C II], and CO line ratios, and the constraints of the host galaxy dynamical mass in Section 4 , and summarize the 
results in Section 5. A $\Lambda$-CDM cosmology with $H_{0}=71 \mathrm{~km} \mathrm{~s}^{-1} \mathrm{Mpc}^{-1}, \Omega_{M}=0.27$, and $\Omega_{\Lambda}=0.73$ is adopted throughout this paper (Spergel et al. 2007).

\section{OBSERVATIONS}

\subsection{Plateau de Bure Interferometer (PdBI) Observations}

We observed the [C II] $158 \mu \mathrm{m}\left({ }^{2} P_{3 / 2}-{ }^{2} P_{1 / 2}\right)$ and molecular $\mathrm{CO}(6-5)$ line emission from $\mathrm{J} 0100+2802$ using the PdBI. The observations were carried out during summer 2014 in the Dconfiguration using five antennas. We used the WideX wideband correlator in dual polarization with a total bandwidth of $3.6 \mathrm{GHz}$. We setup the correlator centered at the frequency of 260.35 GHz for the [C II] line and $94.72 \mathrm{GHz}$ for the $\mathrm{CO}(6-5)$ line. This corresponds to the quasar $\mathrm{Mg}$ II line redshift of $z=6.30(\mathrm{Wu}$ et al. 2015). The flux density scale was determined based on observations of the standard flux density calibrator MWC349, and the typical calibration uncertainties are $10 \%$ at $3 \mathrm{~mm}$ and $20 \%$ at $1 \mathrm{~mm}$. The phase was checked about every 22 minutes by observing a nearby phase calibrator, J0112+321. We reduced the data using the Grenoble Image and Line Data Analysis System (GILDAS) software (Guilloteau \& Lucas 2000). The maps were made using natural weighting. The FWHM synthesized beam sizes are 2 ". $00 \times 1$ ". 66 and 5!" $41 \times 4$ ". 31 at the $[\mathrm{C} \mathrm{II]}$ and $\mathrm{CO}(6-5)$ frequencies, respectively. The final rms sensitivity of the [C II] line observation is $2.3 \mathrm{mJy}^{\text {beam }}{ }^{-1}$ per $46 \mathrm{~km} \mathrm{~s}^{-1}$ channel, with a total on-source integration time of $4.5 \mathrm{hr}$, and the rms of the $\mathrm{CO}(6-5)$ observation is $0.42 \mathrm{mJy}^{\text {beam }}{ }^{-1}$ per $63 \mathrm{~km} \mathrm{~s}^{-1}$ channel in $13.2 \mathrm{hr}$.

\subsection{VLA Observations}

We observed the $\mathrm{CO}(2-1)$ line emission with the Karl G. Jansky Very Large Array (VLA) in the $K a$-Band $(\sim 32 \mathrm{GHz})$ in C-configuration during 2014 October-November, and the $3 \mathrm{GHz}$ radio continuum in the $S$-band in A-configuration during 2015 June. The observations were performed using the WIDAR correlator with the 8-bit sampler in dual polarization. The setup covers a total bandwidth of $2 \mathrm{GHz}$, with $16128 \mathrm{MHz}$ spectral windows and the channel width in each spectral window is $2 \mathrm{MHz}$. We have spent a total on-source observing time of $7.2 \mathrm{hr}$ for the $\mathrm{CO}(2-1)$ line observation and $2.1 \mathrm{hr}$ for the $3 \mathrm{GHz}$ continuum. The flux density calibration was carried out using the standard VLA calibrator 3C48, with typical calibration uncertainties better than $5 \%$. The phases were checked with nearby point sources.

We reduced the data using the Common Astronomy Software Applications package (CASA v4.4) and the VLA calibration pipeline version 1.3.4. The FWHM synthesized beam size is 0 . $74 \times 0$." 68 at $32 \mathrm{GHz}$ and 0. " $65 \times 0$ " 54 at $3 \mathrm{GHz}$, using robust weighting. For the $\mathrm{CO}(2-1)$ line observations, we binned the data to a channel width of $57 \mathrm{~km} \mathrm{~s}^{-1}$, and obtained a typical $1 \sigma \mathrm{rms}$ noise of $0.067 \mathrm{mJy}^{-1}$ beam $^{-1}$ per channel. The channels affected by radio frequency interference were removed in the $S$-Band observations, resulting in a useful frequency range of $\sim 2.4$ to $4 \mathrm{GHz}$ and a bandwidth of about 1.6 GHz. The final continuum sensitivity at $3 \mathrm{GHz}$ was $3.1 \mu \mathrm{Jy}^{\text {beam }^{-1} \text {. }}$
Table 1

Line and Continuum Properties of J0100+2802

\begin{tabular}{|c|c|}
\hline 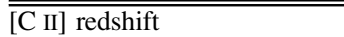 & $6.3258 \pm 0.0010$ \\
\hline [C II] FWHM $\left(\mathrm{km} \mathrm{s}^{-1}\right)$ & $300 \pm 77$ \\
\hline [C II] line flux $\left(\mathrm{Jy} \mathrm{km} \mathrm{s}^{-1}\right)$ & $3.36 \pm 0.46$ \\
\hline \multicolumn{2}{|l|}{ [C II] line luminosity: } \\
\hline$\left(L_{\odot}\right)$ & $(3.56 \pm 0.49) \times 10^{9}$ \\
\hline$\left(\mathrm{K} \mathrm{km} \mathrm{s}^{-1} \mathrm{pc}^{2}\right)$ & $(1.62 \pm 0.22) \times 10^{10}$ \\
\hline $\mathrm{CO}(6-5)$ redshift & $6.3264 \pm 0.0024$ \\
\hline $\mathrm{CO}(6-5) \mathrm{FWHM}\left(\mathrm{km} \mathrm{s}^{-1}\right)$ & $498 \pm 225$ \\
\hline $\mathrm{CO}(6-5)$ line flux $\left(\mathrm{Jy} \mathrm{km} \mathrm{s}^{-1}\right)$ & $0.32 \pm 0.084$ \\
\hline \multicolumn{2}{|l|}{$\mathrm{CO}(6-5)$ line luminosity: } \\
\hline$\left(L_{\odot}\right)$ & $(1.23 \pm 0.32) \times 10^{8}$ \\
\hline$\left(\mathrm{K} \mathrm{km} \mathrm{s}^{-1} \mathrm{pc}^{2}\right)$ & $(1.17 \pm 0.30) \times 10^{10}$ \\
\hline $\mathrm{CO}(2-1)$ line flux $\left(\mathrm{Jy} \mathrm{km} \mathrm{s}^{-1}\right)$ & $0.038 \pm 0.013$ \\
\hline \multicolumn{2}{|l|}{$\mathrm{CO}(2-1)$ line luminosity: } \\
\hline$\left(L_{\odot}\right)$ & $(4.89 \pm 1.67) \times 10^{6}$ \\
\hline$\left(\mathrm{K} \mathrm{km} \mathrm{s}^{-1} \mathrm{pc}^{2}\right)$ & $(1.25 \pm 0.43) \times 10^{10}$ \\
\hline \multicolumn{2}{|c|}{$\mathrm{CO}(1-0)$ line luminosity (derived from the $\mathrm{CO}(2-1)$ line): } \\
\hline$\left(L_{\odot}\right)$ & $(6.11 \pm 2.09) \times 10^{5}$ \\
\hline$\left(\mathrm{K} \mathrm{km} \mathrm{s}^{-1} \mathrm{pc}^{2}\right)$ & $(1.25 \pm 0.43) \times 10^{10}$ \\
\hline $666 \mathrm{GHz}$ continuum (mJy) & $<30$ \\
\hline $353 \mathrm{GHz}$ continuum (mJy) & $4.1 \pm 1.2$ \\
\hline $260 \mathrm{GHz}$ continuum (mJy) & $1.35 \pm 0.25$ \\
\hline $94.5 \mathrm{GHz}$ continuum (mJy) & $<0.1$ \\
\hline $32 \mathrm{GHz}$ continuum $(\mu \mathrm{Jy})$ & $14.8 \pm 4.3$ \\
\hline $3 \mathrm{GHz}$ continuum $(\mu \mathrm{Jy})$ & $104.5 \pm 3.1$ \\
\hline \multicolumn{2}{|l|}{ FIR luminosity $\left(L_{\odot}\right)$ : } \\
\hline$T_{\text {dust }}=47 \mathrm{~K}, \beta=1.6$ & $(3.5 \pm 0.7) \times 10^{12}$ \\
\hline$T_{\text {dust }}=40 \mathrm{~K}, \beta=1.6$ & $(2.4 \pm 0.5) \times 10^{12}$ \\
\hline$T_{\text {dust }}=60 \mathrm{~K}, \beta=1.6$ & $(6.0 \pm 1.2) \times 10^{12}$ \\
\hline \multicolumn{2}{|l|}{ dust mass $\left(M_{\odot}\right)$ : } \\
\hline$T_{\text {dust }}=47 \mathrm{~K}, \beta=1.6$ & $(2.0 \pm 0.4) \times 10^{8}$ \\
\hline$T_{\text {dust }}=40 \mathrm{~K}, \beta=1.6$ & $(3.1 \pm 0.6) \times 10^{8}$ \\
\hline$T_{\text {dust }}=60 \mathrm{~K}, \beta=1.6$ & $(1.1 \pm 0.2) \times 10^{8}$ \\
\hline
\end{tabular}

\subsection{SCUBA-2 Observations}

We observed the $450 \mu \mathrm{m}(666 \mathrm{GHz})$ and $850 \mu \mathrm{m}(353 \mathrm{GHz})$ dust continuum from $\mathrm{J} 0100+2802$ using the Submillimetre Common-User Bolometer Array 2 (SCUBA-2; Holland et al. 2013) on the James Clerk Maxwell Telescope (JCMT) during 2015 November. The observations were carried out in Band 2 weather conditions (i.e., $0.05<\tau_{225 \mathrm{GHz}}<0.008$ ), and in "CV DAISY" mode, which is designed for point/compact source observations. The beam size of SCUBA-2 is 7!" 9 at $666 \mathrm{GHz}$ and $13^{\prime \prime}$ at $353 \mathrm{GHz}$. We observed the target in four 30 minute scans with a total on-source time of $\sim 2 \mathrm{hr}$. The data was reduced using the STARLINK SCUBA-2 pipeline for faint point sources (Chapin et al. 2013), and we obtained a $1 \sigma$ pointsource sensitivity of $1.2 \mathrm{mJy}$ at $353 \mathrm{GHz}$ and $10 \mathrm{mJy}$ at $666 \mathrm{GHz}$.

\section{RESULTS}

We summarize the measurements of redshift, line flux, FWHM line width, and luminosity of the [C II], $\mathrm{CO}(6-5)$, and $\mathrm{CO}(2-1)$ lines, as well as the continuum in Table 1 . The [C II] and $\mathrm{CO}(6-5)$ lines are clearly detected (Figure 1), as well as the continuum emission at 353,260 , and $3 \mathrm{GHz}$. We fit a Gaussian line profile to the $[\mathrm{C} \mathrm{II}]$ and $\mathrm{CO}(6-5)$ line spectra to determine the redshifts and line widths, and integrate the continuumsubtracted data over the line-emitting channels to get the line flux. The redshifts and line widths measured on the [C II] and 

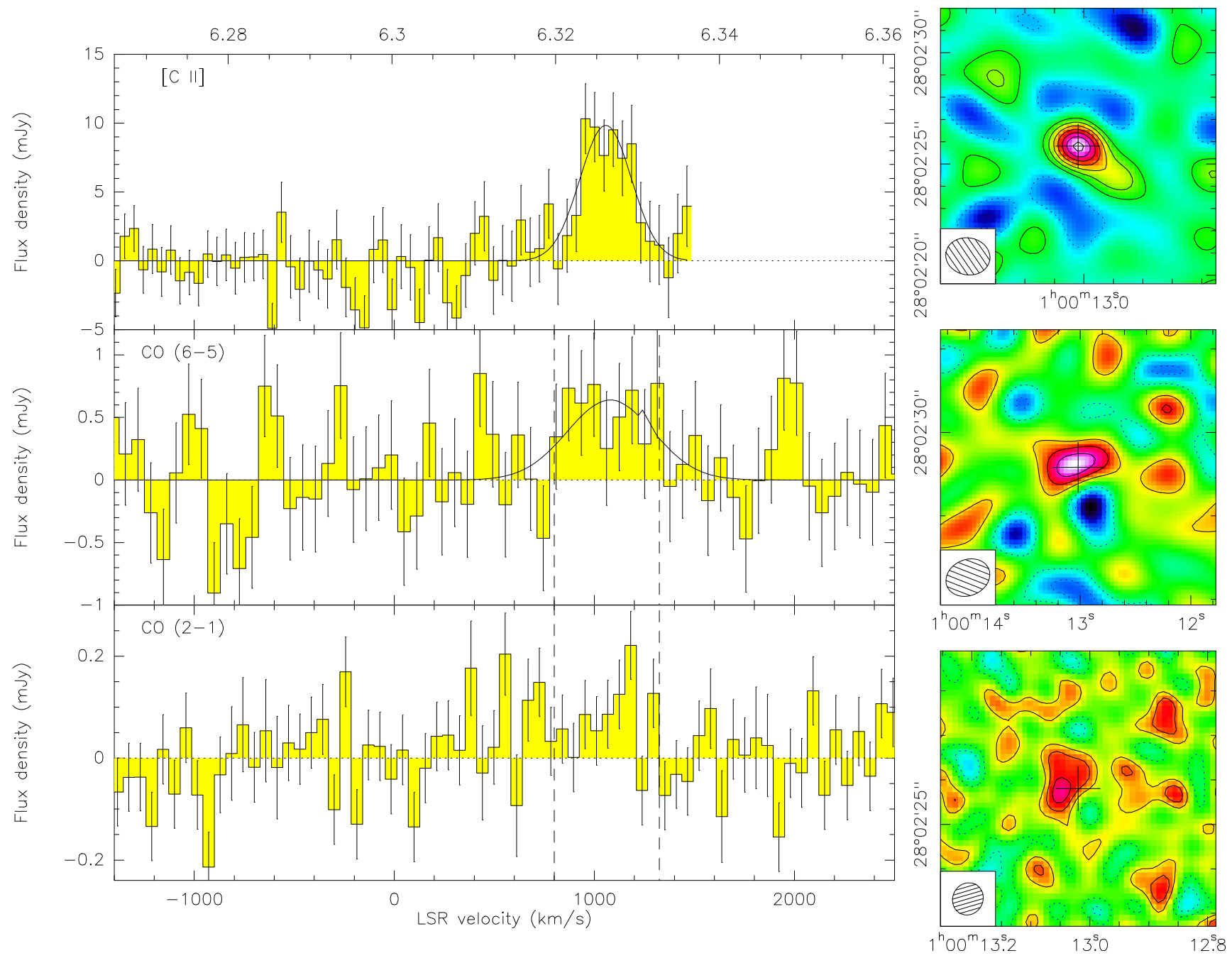

Figure 1. Line spectra (left) and velocity-integrated map (right) of the [C II] $158 \mu \mathrm{m}$ (upper panel), CO(6-5) (middle panel), and CO(2-1) line emission (lower panel) from $\mathrm{J} 0100+2802$. The bottom abscissa shows the radio velocity in Kinematic Local Standard of Rest (LSRK) frame, where zero velocity corresponds to $z_{\mathrm{Mg}}$ II $=6.30$ (Wu et al. 2015). The top abscissa denotes the corresponding redshift. The contours for the [C II] line intensity map are [-2, $-1,1,2,3,4,5,6,7]$ $\times 0.77 \mathrm{mJy}$ beam $^{-1}$, and $[-2,-1,1,2,3] \times 0.15 \mathrm{mJy} \mathrm{beam}^{-1}$ for the $\mathrm{CO}(6-5)$ line. The $\mathrm{CO}(2-1)$ line intensity map is obtained by integrating over the velocity range defined by the $\mathrm{CO}(6-5)$ line (vertical dashed lines), and contours are $[-2,-1,1,2,3] \times 23 \mu \mathrm{Jy}_{\text {beam }}^{-1}$. The cross in each panel denotes the position of the optical quasar, and the synthesized beams are plotted at the bottom left of each map.

$\mathrm{CO}(6-5)$ lines are consistent with each other within the errors, and we adopt $z_{[\mathrm{C} \mathrm{II]}}=6.3258 \pm 0.0010$ as the redshift of the quasar host galaxy. We obtain tentative detections for the $\mathrm{CO}(2-1)$ line and the $32 \mathrm{GHz}$ continuum. The central frequency and width of the $\mathrm{CO}(2-1)$ line are difficult to constrain due to the poor signal-to-noise ratio $(\mathrm{S} / \mathrm{N})$ of the spectrum. We integrate the visibility data over the velocity range of the $\mathrm{CO}(6-$ 5) line emission (i.e., $800 \mathrm{~km} \mathrm{~s}^{-1}$ to $1300 \mathrm{~km} \mathrm{~s}^{-1}$ in Figure 1), and the intensity map shows a $3.6 \sigma$ peak about 0 ". 27 away from the optical quasar position. Considering the measurement uncertainties and the astrometric mismatch between the radio and optical frames, this is consistent with the quasar optical position. We also measure the $32 \mathrm{GHz}$ continuum emission to be $S_{32 \mathrm{GHz}}=14.8 \pm 4.3 \mu \mathrm{Jy}$ by averaging all the line-free spectral windows. We subtract the continuum from the lineemitting channels, resulting in a $\mathrm{CO}(2-1)$ line flux of $0.038 \pm 0.013 \mathrm{Jy} \mathrm{km} \mathrm{s}^{-1}$ at the peak position. Due to the poor $\mathrm{S} / \mathrm{N}$ of the intensity map, we cannot obtain reliable measurement of the source size or address if there are more extended components with lower surface brightness.
The quasar Mg II line emission from $\mathrm{J} 0100+2802$ measures a redshift of $z_{\mathrm{Mg} \text { II }}=6.301 \pm 0.006$ (Figure 3 in $\mathrm{Wu}$ et al. 2015), which is blueshifted by $1020 \pm 250 \mathrm{~km} \mathrm{~s}^{-1}$ compared to $z_{[\mathrm{C} \mathrm{II}]}$. Similar blueshifted Mg II lines with velocity offsets of a few hundred to $1700 \mathrm{~km} \mathrm{~s}^{-1}$ are also detected in several other $z>6$ quasars (Willott et al. 2015; Venemans et al. 2016). Such large Mg II line velocity offsets with respect to the quasar host galaxy redshift are rare at low redshift (Richards et al. 2002), and may suggest outflowing gas in the broad line region at an early evolutionary phase. A larger sample of high- $z$ quasars with $z_{\mathrm{Mg} \text { II }}$ and $z_{[\mathrm{C} \text { II] }}$ measurements is required to investigate the relationship between $\mathrm{Mg}$ II velocity offset and quasar luminosities (Venemans et al. 2016) and address the origin of the blueshifted quasar $\mathrm{Mg}$ II line.

\section{DISCUSSION}

\subsection{Continuum Emission and SED}

The new observations we present here measure the restframe FIR to radio continuum emission from J0100+2802. We 


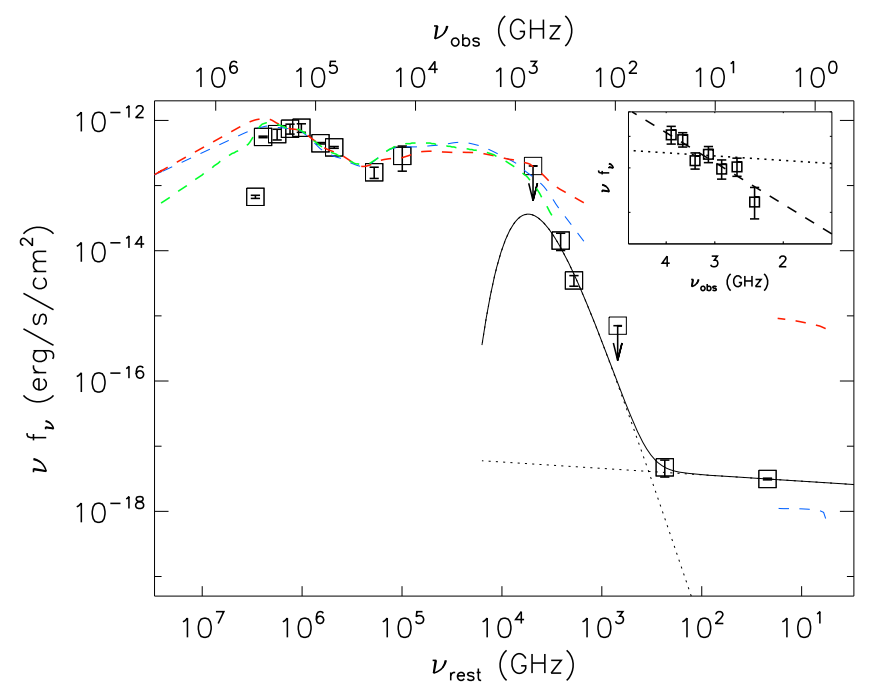

Figure 2. Rest-frame UV-to-radio SED of J0100+2802. The squares show the data from SDSS, 2MASS, and WISE (Wu et al. 2015), and the new measurements in this work. The arrows denote $3 \sigma$ upper limits. The templates of local radio-quiet (blue line) and radio-loud quasars (red line, Elvis et al. 1994) and SDSS optically luminous quasars (green line, Richards et al. 2006) are plotted and scaled to the 2 MASS $H$-band flux. The dotted lines are the graybody and power-law (with $f_{\nu} \sim \nu^{\alpha}$ and $\alpha_{22 \mathrm{GHz}}^{234 \mathrm{GHz}}=-0.90$ ) fit to the FIR and radio continuum, respectively, and the black solid line shows the total emission from these two components. The inner panel shows the $S$-band continuum fluxes measured in every $258 \mathrm{MHz}$ frequency bins from 2.3 to $4 \mathrm{GHz}$. The dotted line is the power law with $\alpha_{22}^{234 \mathrm{GHz}}=-0.90$ fitted to the $K a$-band continuum and the $S$-band flux density averaged over the total $1.6 \mathrm{GHz}$ bandwidth, while the dashed line is the best-fitting result to only the $S$ band data with $\alpha_{22 \mathrm{GHz}}=-0.06$.

plot the SED of J0100+2802 in Figure 2 (Wu et al. 2015), together with the data from SDSS, the Two Micron All Sky Survey (2MASS), and the Wide-field Infrared Survey Explorer (WISE). The templates of optically luminous quasars from Elvis et al. (1994) and Richards et al. (2006) are also plotted for comparison. We scale the templates to the 2MASS $H$-band flux, and the (sub)mm measurements show no excess compared to the FIR emission of the scaled templates. We cannot rule out that the FIR continuum emission from $\mathrm{J} 0100+2802$ is dominated by thermal emission from the active galactic nucleus (AGN)-heated dust. However, the detections of [C II] and $\mathrm{CO}$ line emission do argue for a star-forming interstellar medium (ISM) in the nuclear region (see Section 4.2). We fit the (sub)mm flux densities to an optically thin graybody model with a dust temperature of $T_{\text {dust }}=47 \mathrm{~K}$ and an emissivity index of $\beta=1.6$ from the (sub)mm-detected quasars at $z>1.7$ (Beelen et al. 2006). This results in a FIR $(42.5-122.5 \mu \mathrm{m})$ luminosity of $L_{\mathrm{FIR}}=(3.5 \pm 0.7) \times 10^{12} L_{\odot}$, and a $8-1000 \mu \mathrm{m}$ IR luminosity of $L_{\mathrm{IR}}=(5.0 \pm 0.9) \times 10^{12} L_{\odot}$. The star formation rate (SFR) estimated with the IR luminosity using the relation in Kennicutt (1998) is $850 M_{\odot} \mathrm{yr}^{-1}$. As the fraction contributed from the torus is unknown, this constrains the upper limits of the star formation-powered FIR emission and SFR in the host galaxy. The dust mass calculated based on the above dust temperature and emissivity index assumption is $M_{\text {dust }}=(2.0 \pm 0.4) \times 10^{8} M_{\odot}$. We adopt a dust absorption coefficient of $\kappa_{\mathrm{d}}(\lambda) \sim \lambda^{-\beta}$ and $\kappa_{\mathrm{d}}(125 \mu \mathrm{m})=18.75 \mathrm{~cm}^{2} \mathrm{~g}^{-1}$ (Hildebrand 1983) in the calculation. If a higher dust temperature of $60 \mathrm{~K}$ is adopted (i.e., the highest temperature seen among the (sub)mm-detected $z \sim 6$ quasars, Leipski et al. 2013), the FIR luminosity and SFR increase by a factor of 1.7 , and the dust mass will decrease by a factor of 1.8. If we adopt the lowest dust temperature of $40 \mathrm{~K}$, found by Leipski et al. (2013), the FIR and SFR with decrease by a factor of 1.5, and the dust mass increases by a factor of 1.6 (Table 1).

The VLA $K a$ - and $S$-band observations measure the restframe 234 and $22 \mathrm{GHz}$ continuum emission, respectively. The corresponding continuum luminosities are $\nu L_{\nu, 234 \mathrm{GHz}}=$ $(5.8 \pm 1.7) \times 10^{8} L_{\odot}$ and $\nu L_{\nu, 22 \mathrm{GHz}}=(3.9 \pm 0.1) \times 10^{8} L_{\odot}$. We calculate the radio-loudness of this object, adopting the definition of $R=f_{\nu, 5 \mathrm{GHz}} / f_{\nu, 4400 \AA}$ (Kellermann et al. 1989). The rest-frame $4400 \AA$ flux density $\left(f_{\nu, 4400} \AA\right)$ is interpolated using the scaled Richards et al. (2006) optically luminous quasar template (Figure 1), and the rest-frame $5 \mathrm{GHz}$ flux density $\left(f_{\nu, 5 \mathrm{GHz}}\right)$ is extrapolated from $22 \mathrm{GHz}$ data assuming a power-law radio continuum of $f_{\nu} \propto \nu^{\alpha}$. This constrains the radio-loudness to be $R=0.9$ for a steep spectrum of $\alpha=-0.9$, or $R=0.2$ for a flat spectrum of $\alpha=-0.06$ (see below for the discussion of spectral index), indicating that this object is radio-quiet. However, the 234 and $22 \mathrm{GHz}$ luminosities are more than an order of magnitude higher than the thermal bremsstrahlung or nonthermal synchrotron continuum expected from star-forming activities (e.g., Condon 1992; Yun et al. 2000; Yun \& Carilli 2002; Zakamska et al. 2016). For instance, the thermal bremsstrahlung or nonthermal synchrotron continuum estimated with an SFR of $850 M_{\odot} \mathrm{yr}^{-1}$ using the formulae in Yun \& Carilli (2002), contributes $<5 \%$ of the detected continuum emission at both frequencies. Additionally, the thermal dust emission has only a minor contribution of $12 \%-21 \%$ to the $234 \mathrm{GHz}$ flux density, based on the graybody models with $T_{\text {dust }}=60-40 \mathrm{~K}$ described above. Thus, the continuum emission in $\mathrm{J} 0100+2802$ at frequencies of $234 \mathrm{GHz}$ and lower is dominated by the radio activity of the central AGN.

In Figure 1, we adopt the graybody model with $T_{\text {dust }}=47 \mathrm{~K}$ to remove the dust continuum at $234 \mathrm{GHz}$ and fit a power law $\left(f_{\nu} \sim \nu^{\alpha}\right)$ to the remaining $234 \mathrm{GHz}$ flux density and the $22 \mathrm{GHz}$ data. This estimates the $234-22 \mathrm{GHz}$ two-point spectral index to be $\alpha_{22}^{234 \mathrm{GHz}}=-0.90 \pm 0.15$. We also measure the spectral index within the $S$-band by averaging the data in every two $128 \mathrm{MHz}$ spectral windows from the observed frequencies of $2.4-4 \mathrm{GHz}$. In contrast to the steep spectrum indicated by $\alpha_{22}^{234 \mathrm{GHz}}$, the best-result fit to the seven data points in the $S$-band yields a flat spectrum with $\alpha_{22 \mathrm{GHz}}=-0.06 \pm 0.22$. $\mathrm{J} 0100+2802$ provides a great example for studying the radio activity in the most optically luminous and radio-quiet nucleus at the earliest epoch. We will need further observations at multiple radio frequencies to check if the radio continuum is indeed flattened or inverse around $22 \mathrm{GHz}$. Additionally, milli-arcsecond resolution observations through Very Long Baseline interferometry are needed to measure the spatial extent of the radio source in this object and address if there are multiple components contributing to the detected radio continuum, as was widely discussed for the radio-quiet quasars at lower redshift (e.g., Ulvestad et al. 1999, 2005)

\subsection{Luminosity Ratios and Gas Masses}

Based on the FIR luminosity derived in the previous section, we constrain the [C II]-to-FIR luminosity ratio to be $0.0010 \pm 0.0002$. This is comparable to the values found in other [C II]-detected $z>5.7$ quasars that have similar moderate FIR luminosities of $L_{\mathrm{FIR}} \lesssim 1 \times 10^{12} L_{\odot}$ to $\sim 4 \times 10^{12} L_{\odot}$, and 
higher than that from the more FIR luminous objects $\left(L_{\mathrm{FIR}} \geqslant 5 \times 10^{12} L_{\odot}\right.$, Wang et al. 2013), i.e., following the trend of decreasing FIR luminosity with increasing [C II]-toFIR luminosity ratios defined by the high-redshift quasars and starburst galaxies (Maiolino et al. 2009; Riechers et al. 2013; De Breuck et al. 2014; Gullberg et al. 2015; Muñoz \& Oh 2015; Willott et al. 2015; Narayanan \& Krumholz 2016; Venemans et al. 2016). The SFR estimated with the [C II] luminosity is $560 M_{\odot} \mathrm{yr}^{-1}$, adopting the SFR-[C II] luminosity relation for high-redshift galaxies in De Looze et al. (2014). This is consistent with the SFR estimates based on the dust continuum.

The CO observations measure a $\mathrm{CO}$ (6-5)-to-(2-1) line flux ratio of $8.4 \pm 3.6$ and a line luminosity ratio of $L_{\mathrm{CO}(6-5)}^{\prime} / L_{\mathrm{CO}(2-1)}^{\prime}=0.94 \pm 0.40$. This is consistent with the ratios found in other $\mathrm{CO}$-detected $z>5.7$ quasars within the errors (Wang et al. 2011; Stefan et al. 2015), suggesting that the detected CO emission is likely from a highly excited molecular gas component peaked at $J=6$ or higher (Riechers et al. 2009; Gallerani et al. 2014; Stefan et al. 2015). If we assume that the low-J CO transitions are thermalized, i.e., $L_{\mathrm{CO}(1-0)}^{\prime}=L_{\mathrm{CO}(2-1)}^{\prime}$ (e.g., Carilli \& Walter 2013), and adopt a luminosity-to-mass conversion factor of $\alpha_{\mathrm{CO}}=0.8 M_{\odot} / \mathrm{K} \mathrm{km} \mathrm{s}^{-1} \mathrm{pc}^{2}$ from the local ultraluminous infrared galaxies (Downes \& Solomon 1998), the detected $\mathrm{CO}(2-1)$ line flux yields a molecular gas mass of $M_{\text {mol }}=(1.0 \pm 0.3) \times 10^{10} M_{\odot}$. However, the total molecular gas mass in the quasar host could be larger considering that some of the $\mathrm{CO}$ (2-1) line flux from the low surface brightness region and the line wings might be missing due to the poor $\mathrm{S} / \mathrm{N}$ of the observation.

We compare the luminosity ratios of the FIR continuum, [C II], and CO lines from $\mathrm{J} 0100+2802$ to the models of the photodissociation region (PDR ${ }^{13}$; Kaufman et al. 1999, 2006; Pound \& Wolfire 2008) in Figure 3 to investigate the gas density $n$ and the incident far-ultraviolet radiation field $G_{0}$ (in units of the Habing Field, $1.6 \times 10^{-3} \mathrm{ergs} \mathrm{cm}^{-2} \mathrm{~s}^{-1}$ ) of the ISM in the quasar host galaxy. As a one-side illuminated slab geometry was adopted in the model, we here divide the optically thin FIR ${ }^{14}$ and [C II] line emission by a factor of 2 to match the condition in the model. We also multiply $L_{[\mathrm{C} \text { II] }}$ by a factor of 0.7 to estimate and exclude the [C II] emission from the diffuse region (Stacey et al. 1991; Colbert et al. 1999). According to Figure 3, the overlap region of the three luminosity ratios suggests a $G_{0}$ of a few thousand and $n \gtrsim 1 \times 10^{5} \mathrm{~cm}^{-3}$. These are only preliminary constraints on the physical condition of the star-forming region in the host galaxy of $\mathrm{J} 0100+2802$. There are still large uncertainties in the calculation of FIR luminosity. The fractions of [C II] and CO emission from the PDR region are also not well-determined. A larger FIR luminosity with a higher $T_{\text {dust }}$ will result in a higher $G_{0}$, and any contribution from the X-ray dominated regions (XDR) to the detected CO (6-5) line emission (e.g., Gallerani et al. 2014) will result in a higher $L_{[\mathrm{C}}{ }_{\mathrm{II}} / L_{\mathrm{CO}(6-5)}$ and a lower $L_{\mathrm{CO}(6-5)} / L_{\mathrm{CO}(2-1)}$ from the PDR region and move the best-fit gas density to $n<10^{5} \mathrm{~cm}^{-3}$.

We estimate the atomic gas mass within the PDR region from the $[\mathrm{C} \mathrm{II}]$ line luminosity using Equation (1) in HaileyDunsheath et al. (2010), adopting the best-fit parameters

\footnotetext{
13 The line and FIR ratio models are taken from the Photo Dissociation Region Toolbox (http://dustem.astro.umd.edu/pdrt/).

${ }^{14}$ The FIR luminosity is re-calculated in the range of $30-1000 \mu \mathrm{m}$ to match the definition in the models.
}

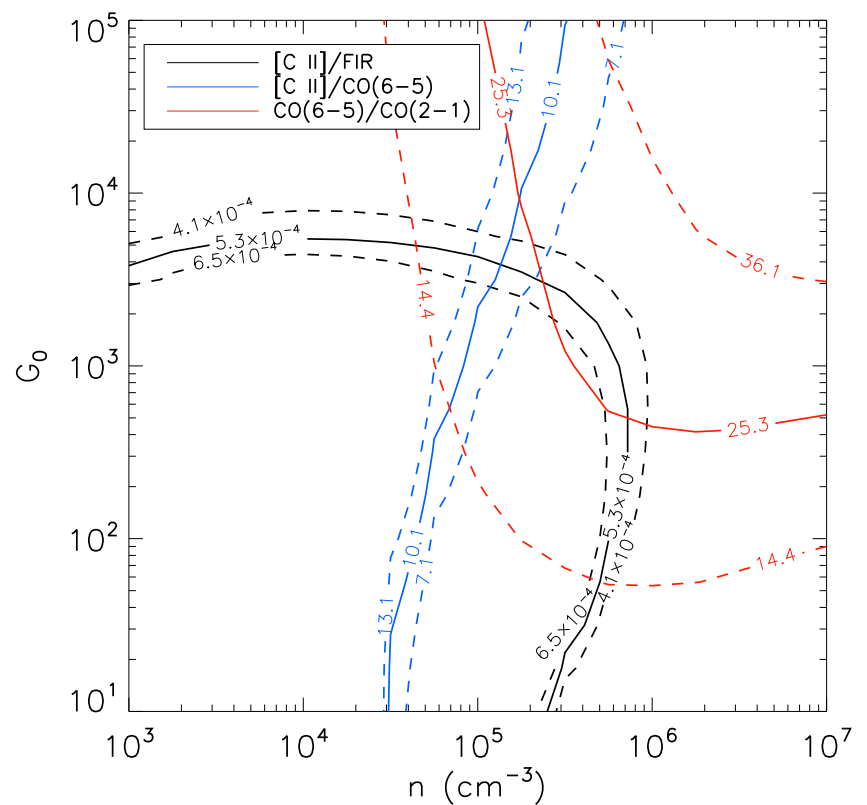

Figure 3. Luminosity ratios of $L_{[\mathrm{C} \mathrm{II}]} / L_{\mathrm{FIR}}$ (black), $L_{[\mathrm{C} \mathrm{I}]} / L_{\mathrm{CO}(6-5)}$ (blue), and $L_{\mathrm{CO}(6-5)} / L_{\mathrm{CO}(2-1)}(\mathrm{red})$ compared to the PDR models of Kaufman et al. (1999) in different conditions of radiation field $G_{0}$ and gas density $n$. Luminosities are all in units of $L_{\odot}$. The dashed contours denote the $\pm 1 \sigma$ error bars from the observing uncertainties. We adopt the optically thin graybody model with $T_{\text {dust }}=47 \mathrm{~K}$ and $\beta=1.6$ to calculate $L_{\mathrm{FIR}}$. The $L_{\mathrm{FIR}}$ here is integrated in the wavelength range of $30-1000 \mu \mathrm{m}$ to match the FIR definition in the model. We also assume that $70 \%$ of the detected [C II] line emission is from the PDR region. Thus the $L_{[\mathrm{C} \mathrm{II}]} / L_{\mathrm{FIR}}$ value in the plot is lower than the value of 0.001 quoted in Section 4.1. We also divide $L_{[\mathrm{C} \mathrm{II}]}$ and $L_{\mathrm{FIR}}$ by a factor of 2 to match the one-sided illuminated geometry in the model.

indicated in Figure 3 (i.e., $G_{0} \sim 4000, n \sim 10^{5.1} \mathrm{~cm}^{-3}$, and a corresponding PDR surface temperature of $T \sim 550 \mathrm{~K}$, Kaufman et al. 1999), a $\mathrm{C}^{+}$abundance of $1.4 \times 10^{-4}$, and a critical density of $n_{\text {crit }}=2.7 \times 10^{3} \mathrm{~cm}^{-3}$. The derived atomic gas mass in the PDR region is $M_{\text {atomic,PDR }} \sim 3 \times 10^{9} M_{\odot}$, which is about $30 \%$ of the molecular gas mass estimated with the $\mathrm{CO}(2-$ 1) line. The mass ratio associated with the detected $\mathrm{CO},[\mathrm{C} \mathrm{II}]$, and FIR dust emission is $\left(M_{\mathrm{mol}}+M_{\text {atomic,PDR }}\right) / M_{\text {dust }} \sim 65$. This is comparable to the mass ratios of other CO-detected quasars and dusty starburst galaxies at high- $z$ (Michalowski et al. 2010; Wang et al. 2010; Riechers et al. 2013).

\subsection{Constraint on Host Galaxy Dynamical Mass}

Among the quasars known at $z>6$, J0100+2802 has the most massive SMBH, with a mass of $(1.24 \pm 0.19) \times 10^{10} M_{\odot}$ (De Rosa et al. 2011; Wu et al. 2015). We use the [C II] line width $\left(\mathrm{FWHM}_{[\mathrm{C} \text { II] }}\right)$ to present a preliminary estimate of the host galaxy mass for this object. We assume that the line is from a rotating gas disk and the circular velocity can be estimated as $v_{\text {cir }}=0.75 \mathrm{FWHM}_{[\mathrm{C} \mathrm{II}] / \sin (i)}$, where $i$ is the inclination angle between the rotation axis of the disk and the line of sight. The [C II] line emission from $\mathrm{J} 0100+2802$ is unresolved in our PdBI observation at $2^{\prime \prime}$ resolution. According to the recent [C II] observations of other $z \sim 6$ quasars at sub-arcsecond resolution, the typical FWHM major axis sizes of the [C II] emission are about $2 \sim 4 \mathrm{kpc}$ (Wang et al. 2013; Willott et al. 2013, 2015; Venemans et al. 2016). Thus, we here estimate the FWHM [C II] source size for J0100+2802 to be $3 \pm 1 \mathrm{kpc}$ (see also Willott et al. 2015; Venemans et al. 2016) and set the diameter of the gas disk $D$ as $1.5 \times$ the FWHM [C II] 


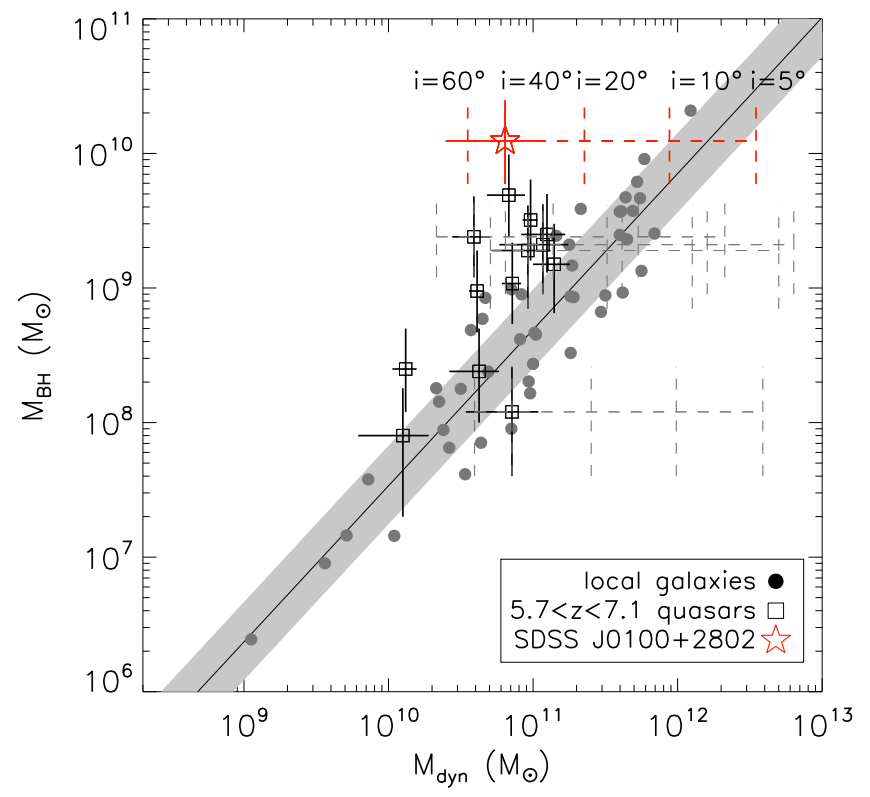

Figure 4. $M_{\mathrm{BH}}$ vs. $M_{\mathrm{dyn}}$ of the $\left[\mathrm{C}\right.$ II]-detected $z>5.7$ quasars. The $M_{\mathrm{dyn}}$ for $z>5.7$ quasars are estimated based on [C II] observations, except for one object, SDSS J114816.64 +525150.3 at $z=6.42$, in which the [C II]-emitting gas at $>1.5 \mathrm{kpc}$ scale is turbulent and the $\mathrm{CO}$ size is adopted (Riechers et al. 2009; Wang et al. 2013; Willott et al. 2013, 2015; Cicone et al. 2015; Venemans et al. 2016). The red star shows $\mathrm{J} 0100+2802$ in this work. For objects that do not have an inclination angle estimated with the resolved [C II] image, we show $M_{\mathrm{dyn}}$ calculated with different inclination angles (dashed lines). The solid line and the gray region show the local relationship with \pm 0.3 dex intrinsic scatter. The gray circles are the sample of local galaxies (Kormendy \& Ho 2013).

source size, i.e., $D=4.5 \pm 1.5 \mathrm{kpc}$. The host galaxy dynamical mass is then $M_{\text {dyn }}\left(M_{\odot}\right)=1.16 \times 10^{5} v_{\text {cir }}{ }^{2} D=$ $[2.6(D / 4.5 \mathrm{kpc}) \pm 1.6] \times 10^{10} / \sin ^{2}(i) M_{\odot}$. The error includes the uncertainties from both the line width and the assumed disk size.

We plot $M_{\mathrm{BH}}$ versus $M_{\mathrm{dyn}}$ for $\mathrm{J} 0100+2802$ and other $z>5.7$ quasars in Figure 4 (Wang et al. 2013; Willott et al. 2013, 2015; Bañados et al. 2015; Venemans et al. 2016), comparing to the SMBH-to-bulge mass relation of local galaxies from Kormendy \& Ho (2013), i.e., $M_{\mathrm{BH}} /\left(10^{9} M_{\odot}\right)=$ $0.49\left(M_{\text {bulge }} / 10^{11} M_{\odot}\right)^{1.16}$. For $\mathrm{J} 0100+2802$ as well as other $z>5.7$ quasars that have SMBH mass measurements based on the quasar Mg II line emission (De Rosa et al. 2011, 2014; Willott et al. 2013, 2015), we follow Willott et al. (2015) and add a 0.3 dex uncertainty to the error bar of the SMBH mass to account for the scatter of the calibration (Shen et al. 2008). For the sample from Wang et al. (2013), which does not have SMBH mass measurements, we adopt the relationship between the $1450 \AA$ luminosity and the quasar bolometric luminosity from Venemans et al. (2016), and calculate the Eddington luminosities and $\mathrm{SMBH}$ masses assuming a typical Eddington ratio and a scatter of $\log \left(L_{\text {bol }} / L_{\text {Edd }}\right)=-0.3 \pm 0.3$ from De Rosa et al. (2011). The $M_{\text {dyn }}$ for most of the $z>5.7$ quasars are estimated based on [C II] observations (Wang et al. 2013; Willott et al. 2013, 2015; Bañados et al. 2015; Venemans et al. 2016). The only exception is the $z=6.42$ quasar SDSS $\mathrm{J} 114816.64+525150.3$, in which the [C II]-emitting gas at $>1.5 \mathrm{kpc}$ scale is turbulent (Cicone et al. 2015) and the CO size is used (Riechers et al. 2009; Stefan et al. 2015) in the $M_{\text {dyn }}$ calculation. According to Figure 4, for any inclination angle of $i \geqslant 10^{\circ}, \mathrm{J} 0100+2802$ is above the local $M_{\mathrm{BH}}-M_{\text {bulge }}$ relation and the \pm 0.3 dex area of the intrinsic scatter (i.e., the gray area in Figure 3). As was discussed in Willott et al. (2015), most of the $z \sim 6$ quasars with SMBH masses on the order of $10^{8} M_{\odot}$ are close to the trend of local galaxies, while the more luminous and massive objects tend to be above this trend (see also Venemans et al. 2016). This suggests that the SMBH grows faster than the quasar host galaxies in these most massive systems at the earliest epoch, unless all these $M_{\mathrm{BH}}>10^{9} M_{\odot}$ quasars are close to face-on. However, as there is no resolved image for $\mathrm{J} 0100+2802$ yet, we do not rule out the possibilities that the gas is unvirialized and the [C II] line width cannot probe the disk circular velocity.

\section{SUMMARY}

We detected [C II], CO, and (sub)mm and radio continuum emission in the host galaxy of the quasar J0100+2802, which hosts the most massive SMBH known at $z \geqslant 6$. The detections probe the properties of the young quasar host at an early evolutionary stage: the (sub)mm continuum indicates moderate FIR emission and constrains the SFR to be $\leqslant 850 M_{\odot} \mathrm{yr}^{-1}$. The $\mathrm{CO}$ and $[\mathrm{C} \mathrm{II}]$ lines estimate the gas mass and gas-to-dust mass ratio that are within the range of other millimeter-detected quasars at $z \sim 6$. The [C II]-to-FIR luminosity ratio J0100 +2802 is higher than that of the most FIR luminous quasars at $z>5.7$, i.e., following the trend of increasing $L_{[\mathrm{C} \mathrm{II}]} / L_{\mathrm{FIR}}$ with decreasing $L_{F I R}$ found for high- $z$ quasars and star-forming systems. The quasar Mg II line emission detected in previous near-infrared spectroscopic observations (Wu et al. 2015) is blueshifted by about $1000 \mathrm{~km} \mathrm{~s}^{-1}$ compared to the host galaxy redshift measured by the [C II] and $\mathrm{CO}$ lines. The host dynamical mass estimated with the [C II] line width suggests that the SMBH is likely to be overmassive, compared to the local relation, though further constraints on the gas kinematics and disk inclination angle are still required.

The data presented in this paper are based on observations under project number S14CY with the IRAM Plateau de Bure Interferometer, projects $14 \mathrm{~B} 151$ and $15 \mathrm{~A} 494$ with the VLA, and project M15BI055 with JCMT/SCUBA-2. IRAM is supported by INSU/CNRS (France), MPG (Germany), and IGN (Spain). The National Radio Astronomy Observatory is a facility of the National Science Foundation operated under a cooperative agreement by Associated Universities, Inc. The James Clerk Maxwell Telescope is operated by the East Asian Observatory on behalf of The National Astronomical Observatory of Japan, Academia Sinica Institute of Astronomy and Astrophysics, the Korea Astronomy and Space Science Institute, the National Astronomical Observatories of China, and the Chinese Academy of Sciences (grant No. XDB09000000), with additional funding support from the Science and Technology Facilities Council of the United Kingdom and participating universities in the United Kingdom and Canada. We are thankful for support from the National Science Foundation of China (NSFC) grants No.11373008 and 11533001, the Strategic Priority Research Program "The Emergence of Cosmological Structures" of the Chinese Academy of Sciences, grant No. XDB09000000, the National Key Basic Research Program of China 2014CB845700, and the Ministry of Science and Technology of China under grant 2016YFA0400703. R. W. acknowledges support from the Thousand Youth Talents Program of China, the NSFC grants 
No. 11443002 and 11473004. X. F. acknowledges support from NSF Grants AST 11-07682 and 15-15115. We thank M. Wolfire for providing the line ratio maps used in the PDRToolBox.

Facilities: IRAM (PdBI), VLA, JCMT (SCUBA-2).

\section{REFERENCES}

Bañados, E., Decarli, R., Walter, F., et al. 2015, ApJL, 805, 8 Bañados, E., Venemans, B. P., Morganson, E., et al. 2014, AJ, 148, 14 Beelen, A., Cox, P., Bertoldi, F., et al. 2006, ApJ, 642, 694

Bertoldi, F., Cox, P., Neri, R., et al. 2003, A\&A, 409, L47 Carilli, C. L., Neri, R., Wang, R., et al. 2007, ApJL, 666, L9

Carilli, C. L., \& Walter, F. 2013, ARA\&A, 51, 105

Chapin, E. L., Berry, D. S., Gibb, A. G., et al. 2013, MNRAS, 430, 2545

Cicone, C., Maiolino, R., Gallerani, S., et al. 2015, A\&A, 574, 14

Colbert, J. W., et al. 1999, ApJ, 511, 721

Condon, J. J. 1992, ARA\&A, 30, 575

De Breuck, C., Williams, R. J., Swinbank, M., et al. 2014, A\&A, 565, 59

De Looze, I., Cormier, D., Lebouteiller, V., et al. 2014, A\&A, 568, 62

De Rosa, G., Decarli, R., Walter, F., et al. 2011, ApJ, 739, 56

De Rosa, G., Venemans, B. P., Decarli, R., et al. 2014, ApJ, 790, 145

Downes, D., \& Solomon, P. M. 1998, ApJ, 507, 615

Elvis, M., Wilkes, B. J., McDowell, J. C., et al. 1994, ApJS, 95, 1

Fan, X., Strauss, M. A., Richards, G. T., et al. 2006, AJ, 131, 1203

Gallerani, S., Ferrara, A., Neri, R., \& Maiolino, R. 2014, MNRAS, 445, 2848

Guilloteau, S., \& Lucas, R. 2000, ASPC, 217, 299

Gullberg, B., De Breuck, C., Vieira, J. D., et al. 2015, MNRAS, 449, 2883

Hailey-Dunsheath, S., Nikola, T., Stacey, G. J., et al. 2010, ApJL, 714, L162

Hildebrand, R. H. 1983, QJRAS, 24, 267

Holland, W. S., Bintley, D., Chapin, E. L., et al. 2013, MNRAS, 430, 2513

Jiang, L., McGreer, I. D., Fan, X., et al. 2015, AJ, 149, 188

Kaufman, M. J., Wolfire, M. G., \& Hollenbach, D. J. 2006, ApJ, 644, 283

Kaufman, M. J., Wolfire, M. G., Hollenbach, D. J., \& Luhman, M. L. 1999, ApJ, 527, 795

Kellermann, K. I., Sramek, R., Schmidt, M., Shaffer, D. B., \& Green, R. 1989, AJ, 98, 1195

Kennicutt, R. C., Jr. 1998, ARA\&A, 36, 189

Kormendy, J., \& Ho, L. C. 2013, ARA\&A, 51, 511
Leipski, C., Meisenheimer, K., Walter, F., et al. 2013, ApJL, 772, 103

Maiolino, R., Caselli, P., Nagao, T., et al. 2009, A\&A, 500, L1

Matsuoka, Y., Onoue, M., Kashikawa, N., et al. 2016, ApJ, submitted (arXiv:1603.02281)

McConnell, N. J., Ma, C.-P., Gebhardt, K., et al. 2011, Natur, 480, 215

Michalowski, M. J., Watson, D., \& Hjorth, J. 2010, ApJ, 712, 942

Muñoz, J. A., \& Oh, S. P. 2015, MNRAS, submitted (arXiv:1510.00397)

Narayanan, D., \& Krumholz, M. 2016, MNRAS, submitted (arXiv:1601. 05803)

Omont, A., Willott, C. J., Beelen, A., et al. 2013, A\&A, 552, A43

Petric, A. O., Carilli, C. L., Bertoldi, F., et al. 2003, AJ, 126, 15

Pound, M. W., \& Wolfire, M. G. 2008, ASPC, 394, 654

Priddey, R. S., Isaak, K. G., McMahon, R. G., et al. 2003, MNRAS, 339,1183

Richards, G. T., Lacy, M., Storrie-Lombardi, L. J., et al. 2006, ApJS, 166, 470

Richards, G. T., Vanden Berk, D. E., Reichard, T. A., et al. 2002, AJ, 124, 1

Riechers, D. A., Bradford, C. M., Clements, D. L., et al. 2013, Natur, 496, 329

Riechers, D. A., Walter, F., Bertoldi, F., et al. 2009, ApJ, 703, 1338

Robson, I., Priddey, R. S., Isaak, K. G., et al. 2004, MNRAS, 351, L29

Shen, Y., Greene, J. E., Strauss, M. A., Richards, G. T., \& Schneider, D. P. 2008, ApJ, 680, 169

Solomon, P. M., \& Vanden Bout, P. A. 2005, ARA\&A, 43, 677

Spergel, D. N., Bean, R., Doré, O., et al. 2007, ApJS, 170, 377

Stacey, G. J., Geis, N., Genzel, R., et al. 1991, ApJ, 373, 423

Stefan, I. I., Carilli, C. L., Wagg, J., et al. 2015, MNRAS, 451, 1713

Thomas, J., Ma, Ch.-P., McConnell, N. J., et al. 2016, Natur, 532, 340

Ulvestad, J. S., Antonucci, R. R. J., \& Barvainis, R. 2005, ApJ, 621, 123

Ulvestad, J. S., Wrobel, J. M., \& Carilli, C. L. 1999, ApJ, 516, 127

Venemans, B. P., Bañados, E., \& Decarli, R. 2015, ApJL, 801, L11

Venemans, B. P., Walter, F., Zschaechner, L., et al. 2016, ApJ, 816, 37

Walter, F., Carilli, C. L., Bertoldi, F., et al. 2004, ApJL, 615, L17

Wang, R., Carilli, C., Neri, R., et al. 2010, ApJ, 714, 699

Wang, R., Wagg, J., Carilli, C., et al. 2011, ApJL, 739, L34

Wang, R., Wagg, J., Carilli, C., et al. 2013, ApJ, 773, 44

Willott, C. J., Bergeron, J., \& Omont, A. 2015, ApJ, 801, 123

Willott, C. J., Delorme, P., Reylé, C., et al. 2010, AJ, 139, 906

Willott, C. J., Omont, A., \& Bergeron, J. 2013, ApJ, 770, 13

Wu, X.-B., Wang, F., Fan, X., et al. 2015, Natur, 518, 512

Yun, M. S., \& Carilli, C. L. 2002, ApJ, 568, 88

Yun, M. S., Carilli, C. L., Kawabe, R., et al. 2000, ApJ, 528, 171

Zakamska, N. L., Lampayan, K., Petric, A., et al. 2016, MNRAS, 455, 4191 